\title{
Predicting whether a material is ductile or brittle
}

\author{
R. P. Thompson*, W. J. Clegg \\ Department of Materials Science and Metallurgy, \\ University of Cambridge, \\ 27 Charles Babbage Road, Cambridge CB3 OFS
}

\begin{abstract}
In this paper we discuss the various models that have been used to predict whether a material will tend to be ductile or brittle. The most widely used is the Pugh ratio, $G / K$, but we also examine the Cauchy pressure as defined by Pettifor, a combined criterion proposed by Niu, the Rice and Thomson model, the Rice model, and the Zhou-Carlsson-Thomson model. We argue that no simple model that works on the basis of simple relations of bulk polycrystalline properties can represent the failure mode of different materials, particularly where geometric effects occur, such as small sample sizes. Instead the processes of flow and fracture must be considered in detail for each material structure, in particular the effects of crystal structure on these processes.
\end{abstract}

Keywords: Ductility, Ductility Criteria, Fracture, Yield, Dislocations, Lattice Resistance, Crystal Structure

\section{Elastic ductility criteria}

\subsection{The Pugh ratio}

Many models exist that try to predict the plastic behaviour of materials. By far the most widely used, no doubt due to the ease with which elastic constants can be measured or calculated by density functional 5 theory, is that of Pugh [1].

The Pugh ratio, $G / K$ where $K$ is the bulk modulus and $G$ is the shear modulus, represents the competition between two processes, plasticity and fracture. If plasticity is easier then a material will tend to be ductile, whereas if fracture is easier then a material will tend to be brittle.

Pugh assumed that the yield stress, i.e. a measure of the difficulty of plastic deformation, scales with the shear modulus, as the Orowan bowing [1] stress is:

$$
\sigma_{y}=\frac{G b}{\lambda}
$$

where $\sigma_{y}$ is the yield stress, $G$ is the shear modulus, $b$ is the Burgers vector, and $\lambda$ is the size of the Frank-Read source [2]. Pugh assumed that $\lambda$ does not vary between metals, i.e. any work hardening can be neglected.

\footnotetext{
* Corresponding author

Email addresses: rpt26@cam.ac.uk (R. P. Thompson), wjc1000@cam.ac.uk (W. J. Clegg)
} 
Pugh associated this with the Brinell hardness number:

$$
\text { B.H.N. }=\frac{G b}{c}
$$

where B.H.N. is the Brinell hardness number and $c$ is a constant for a particular crystal structure, which is not explicitly defined by Pugh, but presumably relates to available slip systems and average Schmid factors.

The fracture stress of a material can also be linked to elastic constants. Pugh noted that fracture stress scales with the Young modulus, $E$ [3], and that the surface energy of a material, to which some of the work of fracture must be converted [4], was shown to correlate with $E$ by Elliott [5]; however Pugh neglects the fact that during the fracture of ductile materials such as aluminium, the energy dissipated by dislocations is much larger than the surface energy. The surface energy, and therefore the stiffness, only has a small influence on the fracture stress of ductile materials.

Pugh suggested that the particular constraints on strain state at the crack tip will cause the relevant elastic modulus to vary between two limiting cases: the Young modulus, $E$, and the bulk modulus, $K$. Since these constants generally scale together, for convenience Pugh used the bulk modulus, and took the fracture stress to obey:

$$
\sigma_{*} \propto K a
$$

where $a$ is a lattice parameter.

If this is true, then the ratio of the yield stress, as characterised by the Brinell hardness, and the fracture stress will be given by the expression:

$$
\frac{B \cdot H \cdot N .}{\sigma_{*}} \propto \frac{G b}{c K a}
$$

As noted by Pugh the crystal structure will affect this criterion, however these are usually neglected to enable easy comparison of materials. If the effects of crystal structure are neglected, $b / a c$ is constant, the relative difficulty of plastic flow and fracture is represented by:

$$
\frac{B \cdot H \cdot N .}{\sigma_{*}} \propto \frac{G}{K} .
$$

Hence the ratio $G / K$ provides a measure of the likely nature of a material's failure: a high value of $G / K$ implies brittle failure, while a low value implies ductile failure. This assumes that the changes in crystal structure affect both processes to the same degree.

Thermal effects were neglected for metals like iron, for which the homologous temperature is low at room temperature ( $\sim 0.16$ for iron). However, it is well documented that the yield stress of iron falls by almost three orders of magnitude between absolute zero and room temperature $[6,7]$ due to the thermally activated nature of the lattice resistance. This is much greater than the change in the shear modulus, which falls by just over $5 \%$ over the same temperature range [8].

Pugh collated a large amount experimental data to support these ideas [9-11]. The yield stress was estimated from the Brinell hardness across a large range of metals with body- and face-centred cubic, and 
hexagonal crystal structures. The data presented are generally consistent, though there are discrepancies, notably $\mathrm{Ca}, \mathrm{Pt}, \mathrm{Be}$, and, perhaps surprisingly given its isotropy, W. These discrepancies are associated with twinning [1] or solute drag $[12,13]$.

The Brinell hardness number is measured using a spherical indenter. This has the complication that the indents are not necessarily self-similar. The hardness therefore depends on the load, $W$ and the diameter of the ball, $D$, and is given by [9]:

$$
\text { B.H.N. }=\frac{2 W}{\pi D^{2}\left[1-\sqrt{1-(d / D)^{2}}\right]}
$$

where $d$ is the diameter of the indent.

The Brinell hardness number of two indents, in the same material, will be the same if the indents are geometrically similar, i.e. if $d / D$ is constant. However there is no indication in Pugh's sources [9-11] that the quoted values were obtained under this condition, so that the experimental support for this idea cannot be conclusive.

This criterion is also often justified by considering the properties of the f.c.c. metals [14]. As shown in Figure 1, soft ductile metals, such as $\mathrm{Au}$ and $\mathrm{Ag}$ have low values of $G / K$, while more brittle metals, particularly Ir, have high values of $G / K$. However despite the fact that Ir is often considered brittle, plastic flow occurs before fracture [15], and the failure mode is strongly dependent on grain boundary properties, particularly impurity levels [16].

\subsection{Cauchy Pressure}

As metals tend to be more ductile than non-metals, another possible criterion is the Cauchy pressure, as discussed by Pettifor [17]. The Cauchy pressure is defined, in terms of the single crystal elastic constants of a cubic material, as $C_{12}-C_{44}$, and can be used to describe the nature of the bonding in a material. A material with a high resistance to bond bending, such as covalently bonded solids, will have a negative Cauchy pressure, i.e. $C_{44}>C_{12}$. In contrast materials with metallic bonding exhibit a positive Cauchy pressure.

It is worth noting that Pettifor states this is a metric for the nature of bonding and appeals to the Pugh ratio when commenting on ductility. It seems likely that the use of the Cauchy pressure as a ductility criterion [18-20] is due to its relative ease of calculation with density functional theory, as with the Pugh ratio. A positive Cauchy pressure is considered to indicate ductile behaviour, while a negative pressure implies brittle behaviour.

55 Niu et al. [18] have shown that one can draw lines where Pettifor's criterion, that the Cauchy pressure should be greater than zero, intersects that of Pugh's criterion at $G / K=0.571$. This is close to the critical value of 0.6, suggested by Gilman [14]. Pugh, himself, does not actually give a critical value for the ratio dividing brittle from ductile materials, although he does state that he expects the transition to be sharp.

It was found that materials followed a broadly hyperbolic trend when the Pugh ratio was plotted against the Cauchy pressure, normalised by the Young modulus [18]. This is plotted for some of the f.c.c. metals 


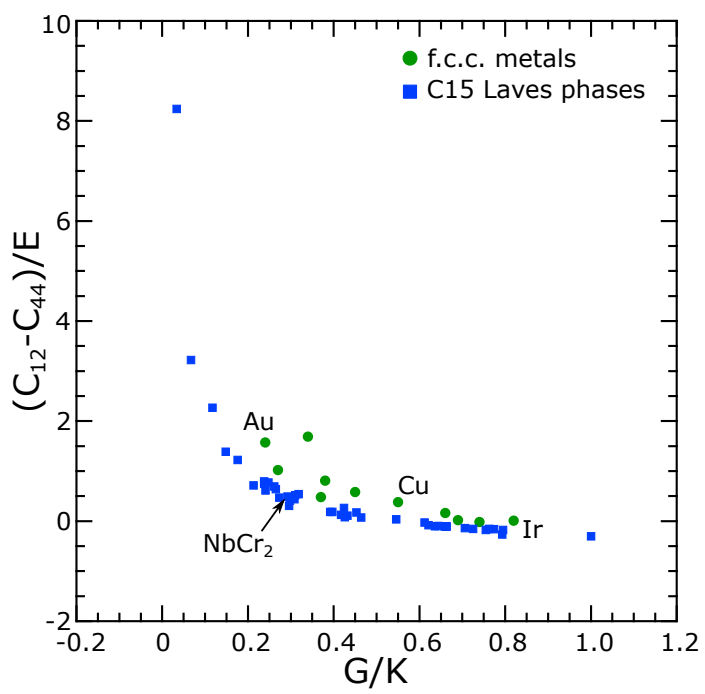

Figure 1: The variation of two ductility criteria, that of Niu et al. [18] and Pugh [1], for the f.c.c. metals and the C15 Laves phases. As can be seen the Laves phases cover a similar range of values for both the criteria and overall fall on the ductile side of the f.c.c. metals. Data from [14, 21-33].

and some of the cubic Laves phases in Figure 1. The Laves phases are describing a different hyperbola to f.c.c. metals, one that is generally lower in $G / K$. Without a theoretical underpinning it is not clear what significance can be attached to this trend.

The low values of $G / K$ and high positive values of the Cauchy pressure in some Laves phases do not correspond to ductile behaviour. While dislocation motion has been observed in micropillars of the Laves phases [34], these phases are brittle [35]. For example the Laves phase $\mathrm{NbCr}_{2}$ has a $\mathrm{Pugh}$ ratio of 0.28 and a normalised Cauchy pressure of 0.56. These values both lie between those of copper and gold, but experimentally the fracture toughness of $\mathrm{NbCr}_{2}$ is found to be around $1.5 \mathrm{MPa} \sqrt{\mathrm{m}}$ to $2 \mathrm{MPa} \sqrt{\mathrm{m}}[24,36]$, which is clearly brittle. This is comparable with ceramics such as borosilicate glass, which have a toughness of about $1.5 \mathrm{MPa} \sqrt{\mathrm{m}}[37]$, and lower than alumina, for which the toughness is approximately $3.5 \mathrm{MPa} \sqrt{\mathrm{m}}$ [38].

In this case, the change of crystal structure has violated the assumptions of the Pugh model. The crystal structure has made the Laves phases brittle by increasing the difficulty of plastic flow, i.e. the yield stress, without a corresponding increase in the difficulty of fracture, i.e. the fracture stress. Since this is an effect of the crystal structure, it cannot be captured by the ratio of elastic constants alone.

Another effect that is often overlooked is that of geometry. In particular the effect of size is known to be significant. For instance, in small micropillars cracking is suppressed and even very brittle materials, such as silicon, will plastically deform [39-41]. The size effect is also significant in indentation where the hardness is known to increase markedly as indent depths become small [42]. As the elastic properties are unchanged, criteria such as the Pugh ratio or the Cauchy pressure cannot account for these phenomena.

At best the Pugh ratio might provide a general indication of behaviour across materials with the same 

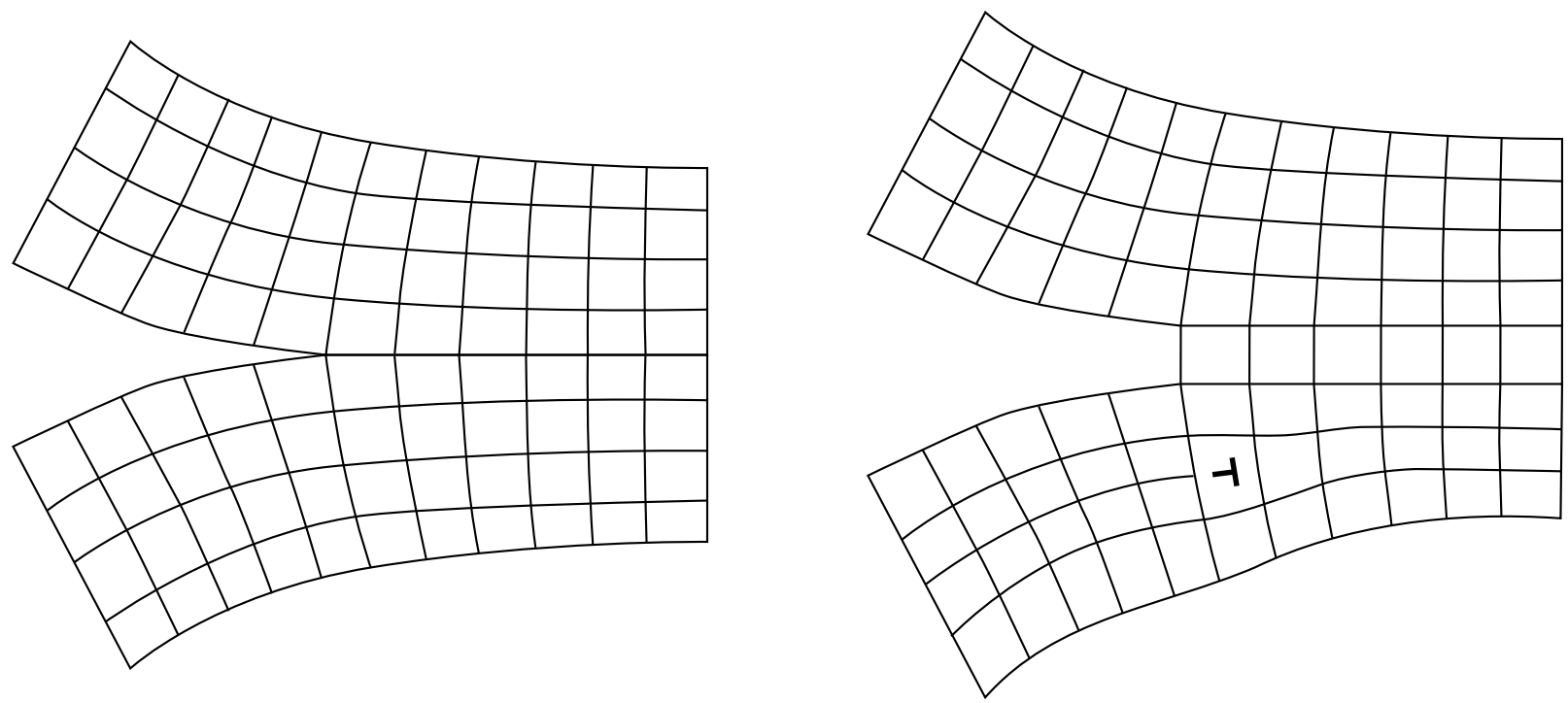

Figure 2: The emission of a dislocation from a sharp crack will cause blunting if there is a component of the Burgers vector normal to the crack plane [43].

or similar crystal structure and deformation mode. Other changes in the balance between the competing processes could prevent even that; for example a change in the nature of dislocations, such as dissociation into partial dislocations. This would not change $G / K$ but would alter the yield stress of the material with no corresponding change in the difficulty of fracture. Certainly the Pugh model and Cauchy pressure cannot provide a basis for the prediction of the behaviour of novel materials.

\section{Dislocation emission criteria}

The other most commonly used models are based on an analysis of the emission of dislocations from a sharp crack tip. Three widely used models are described here but the reasoning is similar. The two competing processes are not fracture and dislocation glide, but instead dislocation nucleation (also termed emission) and fracture.

The emission of a dislocation from the crack tip can cause blunting and dissipate energy, as shown in Figure 2, lowering the stresses at the crack tip and therefore the strain energy release rate. If this process occurs spontaneously then extra work is required to grow the crack and a material will be tougher than if cracks remain atomically sharp.

The exact formulation of the criterion depends on the assumptions made but all depend on an energy barrier experienced by dislocations, usually characterised by $G b$ or $\gamma_{u s}$ where $G$ is the shear modulus, $b$ is the Burgers vector, and $\gamma_{u s}$ is the unstable stacking fault energy. These criteria tend to suffer from either being poorly transferable or being so onerous to calculate that a full treatment of dislocation mobility is more useful. 


\subsection{Rice-Thomson criterion}

Rice and Thomson [43] suggested an approach to characterise whether a material is ductile by considering the balance between fracture and the nucleation of dislocations at the crack tip. This builds on an idea proposed by Kelly et al. [44] that considered the stability of an atomically sharp crack tip and whether crack blunting must occur spontaneously. That study only considered whether it was possible for fracture to occur with no plastic flow at all, even in the region of a sharp crack tip, by considering the ideal shear strength of materials.

Rice and Thomson suggest that the theoretical strength (i.e. an upper bound) would be appropriate for a uniform stress, but, since the shear at the crack tip is localised, actual dislocation processes should be considered.

They consider the forces between a dislocation and a crack tip, which are: first the forces on a dislocation due to the stress field of the crack tip, second the surface tension due to the increased surface area of a blunted crack tip, and third the image force on the dislocation due to the free surface of the crack. The first term drives the dislocation away from the crack tip, the other two attract it. These forces are then used to estimate the activation energy of emitting a blunting dislocation from the crack tip.

They reach an expression for the energy change on emission of a dislocation from a crack tip:

$$
U=G b^{3}\left[r U_{0} \log \frac{r}{\xi_{0}}+U_{l}\left(r-\xi_{0}\right)-\frac{2}{3} U_{s}\left(r^{3 / 2}-\xi_{0}^{3 / 2}\right)\right]
$$

where:

$$
\begin{aligned}
U_{0} & =\frac{2-\nu}{8(1-\nu)}, \\
U_{l} & =2 \frac{\gamma_{s}}{G b} \cos \psi \sin \phi, \\
U_{s} & =\frac{2.092}{\sqrt{1-\nu}} \sqrt{\frac{\gamma_{s}}{G b}} \sin \phi \cos \psi \cos (\phi / 2)
\end{aligned}
$$

and $\nu$ is the Poisson ratio, $\gamma_{s}$ is the surface energy, $\xi_{0}$ is the radius of the dislocation core, $G$ is the shear modulus, $b$ is the Burgers vector, $\psi$ is the angle of the Burgers vector such that $b \cos \psi$ is the component perpendicular to the line defining the crack front, and $\phi$ is the angle of inclination between the crack plane and the slip plane.

The critical condition is therefore:

$$
\frac{d U}{d r}=U_{0} \log \frac{e r}{\xi_{0}}+U_{l}+U_{s} r^{1 / 2}=0 .
$$

Depending on the parameters, the emission may be spontaneous, i.e. no activation energy, or there may be some energy barrier requiring thermal activation. They find that atomically sharp cracks are not stable in f.c.c. metals, which will instead spontaneously emit dislocations, while more brittle materials require thermal activation for the emission of dislocations.

Their derivation suggests that the ratio $G b / \gamma_{s}$ controls the ductile vs brittle nature of materials. Some material data are presented to support this and do correctly rank f.c.c. and b.c.c. metals, some ionic materials, 
and elements with the diamond structure. They propose an approximate condition of $G b / \gamma_{s}>7.5-10$ for sharp cleavage cracks. Interestingly the authors found that iron has an intermediate value between the clearly ductile f.c.c. metals and the clearly brittle ceramic crystals, implying that moderate thermal activation might allow iron alloys to be ductile. This is seen in the ductile-to-brittle transition temperature of iron which is not much below room temperature [45].

\subsection{Rice}

The Rice and Thomson criterion [43] was updated by Rice [46] to be the quotient $\gamma_{u s} / \gamma_{s}$ where $\gamma_{u s}$ is the unstable stacking fault energy and $\gamma_{s}$ is still the surface energy. This criterion follows much the same line of reasoning but employs a Peierls approach to consider the barrier to nucleating a dislocation. A periodic energy is assumed for displacements on a slip plane emanating from the crack tip. There will be a critical load at which local displacements at the crack tip become a dislocation. This obviates the need for the use of a core cut-off radius, as had been used in the original treatment.

The critical strain energy release rate for crack growth is $2 \gamma_{s}$, this can be equated with a critical strain energy release rate for the nucleation of dislocations at the crack tip, as derived by Rice [46]:

$$
G=8 \frac{1+(1-\nu) \tan ^{2} \psi}{(1+\cos \phi) \sin ^{2} \phi} \gamma_{u s}
$$

where the notation matches that for the Rice and Thomson [43] criterion described above, and $\gamma_{u s}$ is the unstable stacking fault energy.

Thus dislocation emission will occur before fracture when:

$$
\frac{\gamma_{s}}{\gamma_{u s}}>4 \frac{1+(1-\nu) \tan ^{2} \psi}{(1+\cos \phi) \sin ^{2} \phi}
$$

This means that the resistance to dislocation nucleation is characterised by $\gamma_{u s}$ rather than $G b$, but otherwise describes much the same competition of processes as the original Rice and Thomson model [43]. Rice compares the predictions with observations and finds the perhaps surprising result that metals such as $\mathrm{Pt}, \mathrm{Al}$ and $\mathrm{Cu}$ are predicted to be close to brittle.

For b.c.c. metals with a crack parallel to a $\{100\}$-type plane with the tip parallel to a $\langle 100\rangle$-type direction intersecting a $\{110\}$ plane on which $\langle 111\rangle$ slip can occur, this gives a condition for dislocation nucleation of $\frac{\gamma_{s}}{\gamma_{u s}}>6.3$ for pure mode I loading. This ratio is dramatically reduced when mode II or III loading is introduced to even modest levels, e.g. $K_{\mathrm{II}}=K_{\mathrm{III}}=0.1 K_{\mathrm{I}}$ nearly halves the necessary ratio to 3.5 [46]. The same analysis for the f.c.c. metals gives a critical ratio of 9.1 for pure mode I loading and 4.2 for the same mixed case. This actually predicts that metals such as $\mathrm{Pt}, \mathrm{Al}$, and $\mathrm{Cu}$ are borderline and so might fail by cleavage at lower temperatures in pure mode I loading, but the shear loading required to prevent this is of the order of $1 \%$. Ni and Ir are the more interesting cases where the amount of shear required is predicted to be larger, and is consistent with with the apparent lack of ductility in Ir [15]. 


\subsection{Zhou-Carlsson-Thomson}

An alternative condition was put forward by Zhou et al. [47] that does not include the surface energy.

They propose the energy to blunt a crack by dislocation emission is dependent on $\gamma_{s}$ in the same way as the energy of growing the sharp crack, since the formation of a dislocation creates ledges and alters the surface area. In this way the ratio of the energies is independent of the surface energy (though the absolute value of either energy is clearly dependent on $\gamma_{s}$ ) and so the crossover from brittle to ductile behaviour is also independent of the surface energy. They find instead that the appropriate quotient is $\gamma_{u s} / G b$ and set a critical value of 0.014. As the authors note, this is an interesting result because the critical value is not the crossover in a competition between two processes, one of fracture and one of plasticity, rather it is a critical value of the Peierls energy.

\subsection{Comparison with data}

An example for which these criteria break down is the contrast between the phases titanium carbide, $\mathrm{TiC}$, and the ternary carbide MAX phase, $\mathrm{Ti}_{3} \mathrm{SiC}_{2}$. The Rice and Thompson model, the Rice model, and the Zhou-Carlsson-Thomson model all correctly predict that $\mathrm{TiC}$ is brittle: the value of $\gamma_{s} / \gamma_{u s}=1.76$ is too small with values in excess of 3 required for ductility $[48,49]$ and the values of $G b / \gamma_{s}=20.48$ and $\gamma_{u s} / G b=0.032$ are too large to indicate ductile behaviour $[49,50]$.

However these same criteria produce similar values for $\mathrm{Ti}_{3} \mathrm{SiC}_{2}$, for which $\gamma_{s} / \gamma_{u s}=1.42, G b / \gamma_{s}=27.3$ 170 and $\gamma_{u s} / G b=0.0219[50,51]$. The Pugh model and the two Rice models $[1,43,46]$ actually predict the MAX phase to be more brittle than stoichiometric titanium carbide. This is at odds with observation, since titanium carbide has a yield stress of over $2 \mathrm{GPa}$ at temperatures below $600^{\circ} \mathrm{C}$ [52] while at room temperature the critical resolved shear strength of $\mathrm{Ti}_{3} \mathrm{SiC}_{2}$ is reported to be $36 \mathrm{MPa}$ [53], though reanalysis of the data presented suggests the strength is somewhat higher at $77 \mathrm{MPa}$ [54].

A key limitation of these two models, highlighted by Rice [46], is that dislocation mobility is not considered, such that cleavage may occur despite the spontaneous emission of dislocations if they are unable to move [55]. Another limit would be if deformation around the crack tip were possible without the nucleation of new dislocations at the crack tip, Rice suggests a high density of very mobile dislocations as one possible scenario in which dislocation nucleation becomes irrelevant.

One drawback of these more physically insightful approaches (at least compared to the essentially empirical Pugh model) is that strictly they apply only for one slip system and one mode of fracture on one plane and so must be recalculated for all possible combinations and then averaged with some appropriate statistical weighting. These criteria can become rather cumbersome: experimental determination of the unstable stacking fault energy and the surface energy is laborious, and calculations quickly become time consuming as combinations of fracture and slip modes are considered.

They also rely in all cases on two assumptions: firstly the energy barrier for slip or emission of dislocations, via the Peierls model, scales linearly with the stacking fault energy or shear modulus, and secondly that the 
stress required for slip scales simply with the Peierls energy. These quantities will be related but it is unlikely that simple relationships exist, as would be required for such ductility criteria to be reliable.

Some studies have considered the Peierls stress, the shear stress required to move a single dislocation through a perfect lattice at absolute zero [66], in layered structures [67-69], but these have usually applied the Peierls result for an isotropic elastic material [66]:

$$
\tau_{p}=\frac{2 G}{1-\nu} \exp \left(-\frac{2 \pi d}{b(1-\nu)}\right)
$$

where $G$ is the shear modulus, $\nu$ is the Poisson ratio, $d$ is the slip plane spacing and $b$ is the Burgers vector. 


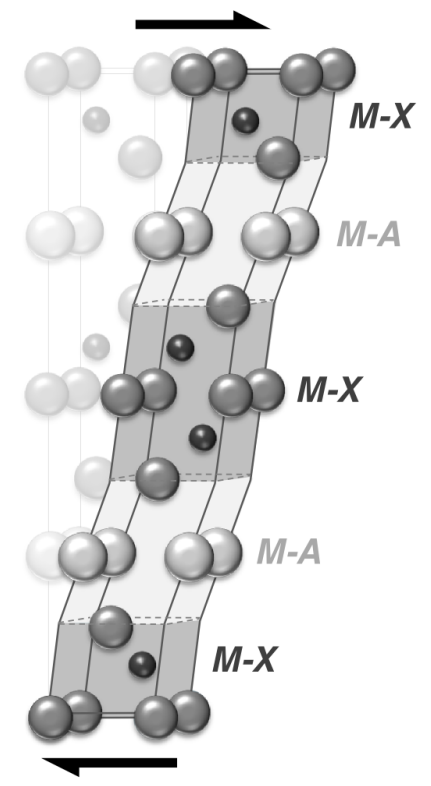

Figure 3: Schematic of heterogeneous strains in a MAX phase unit cell. These strains would arise under the application of a constant stress if the highlighted regions of the unit cell have different shear moduli.

The values of the Peierls stress predicted by Equation 14 for the MAX phases are approximately $0.98 \mathrm{GPa}$ [67]. Values as low as $36 \mathrm{MPa}$ have been reported [53], although a reanalysis suggests the value is closer to $77 \mathrm{MPa}$ [54]. The details of this analysis are given in the appendix. The question therefore arises why the critical resolved shear stress of layered structures is so low. One possibility for the discrepancy, between the standard Peierls expression and what is measured experimentally is that with the effects of elastic inhomogeneity are not normally considered [70].

The poor performance of Peierls models for MAX phases, and potentially for other complex phases, might be due to their treatment as elastically homogeneous. This is surprising because many studies have discussed the heterogeneity of the bonding and electron structure. One recent review was written by Magnuson and Mattesini [71], and discusses the complex and mixed nature of the bonding varying across the different atomic sites: more metallic in the layers containing $\mathrm{M}$ and $\mathrm{A}$ sites, more covalent in the layers containing $\mathrm{M}$ and $\mathrm{X}$ sites, and charge transfer contributing an ionic component to the bonding.

When considered as heterogeneous regions at the sub-unit cell level, clear divides are apparent both chemically, as characterised by the electronegativity, and elastically, as calculated by density functional theory [70]. This heterogeneity of elastic response to an applied stress is shown in Figure 3. Variations in the sub-unit cell moduli with the degree of chemical heterogeneity were identified [70]. These would not be apparent from the macroscopic single crystal elastic constants, let alone the polycrystalline elastic moduli. However they are consistent with those macroscopic properties when combined with a slab model by analogy with composite materials [72].

The effects of this heterogeneity on dislocation motion are substantial given the exponential dependence 


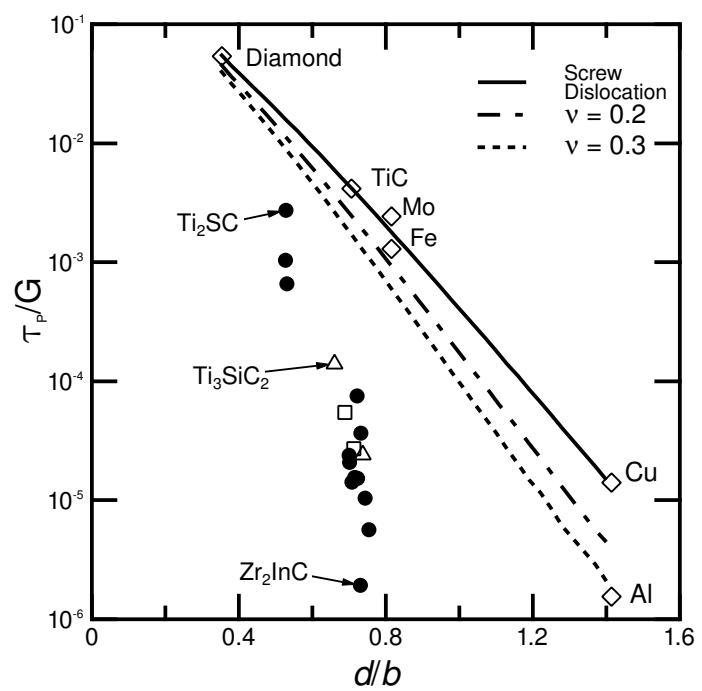

Figure 4: The effects of local heterogeneity on the predicted Peierls stress of the MAX phases compared with the prediction for elastically isotropic and homogeneous phases with the same lattice geometry (i.e. $d / b$, where $d$ is the slip plane spacing and $b$ is the Burgers vector) and some experimental values for simpler crystals. The effects are far stronger than that of the lattice geometry alone and cannot be explained by the bulk properties. From [70].

of the Peierls stress on the elastic properties. If the width is not fixed, as in the case of an isotropic material in equation 14, then the Peierls stress is given by:

$$
\tau_{p}=\frac{2 G}{1-\nu} \exp \left(-\frac{4 \pi w}{b}\right) .
$$

The width can be found numerically by minimising the dislocation energy, if the problem in considered atomistically [70, 73]. Equation 15 shows the sensitivity of the lattice resistance, and thus the yield stress of many non-metallic materials, to the width of a dislocation. The width is strongly affected by the local heterogeneity in the MAX phases. The size of a dislocation is controlled by the competition between two energetic factors; firstly the strain energy in the crystal either side of the slip plane favours a wide dislocation, and secondly the misalignment energy across the slip plane favours a narrow dislocation [74].

In the MAX phases the local heterogeneity allows the introduction of a lower misalignment energy, in the electropositive regions containing $\mathrm{A}$ atoms like $\mathrm{Al}$, and a higher strain energy, in the electronegative regions containing $\mathrm{X}$ atoms like $\mathrm{C}$ or $\mathrm{N}$, than would be expected from the macroscopic properties. This stabilises a wider dislocation core and lowers the lattice resistance. The predicted effects of this heterogeneity on the Peierls stress are shown in Figure 4.

\subsection{Demonstration in a cubic structure: $\mathrm{Ti}_{2} \mathrm{Ni}$}

The previous sections have explained observations of dislocations in the MAX phases, but the question remains as to whether these models of dislocation motion are applicable more broadly. More specifically whether the effect is applicable in systems with a greater, ideally cubic, symmetry to allow full ductility. 
The MAX phases can accommodate chemically, and hence elastically, heterogeneous regions because the unit cells are large, at least parallel the $c$ axis. This suggests that the unit cell of any candidate should be large, of the order of $10 \AA$. The MAX phases show very easy basal slip, but slip out of the basal plane MAX phases [70], as shown in Figure 5.

All the alloys tested were hard, but a significant variation was observed, with the hardness varying between 10.6 and $12.6 \mathrm{GPa}$. The substitution of nickel with cobalt produced the biggest change in hardness, between $\mathrm{Ti}_{2} \mathrm{Ni}$ and $\mathrm{Ti}_{2}$ Co the hardness drops $1.81 \mathrm{GPa}$, or $14.6 \%$, from $12.42 \mathrm{GPa}$ to $10.61 \mathrm{GPa}$, as might be expected

Though more modest in scale than the effects seen in the MAX phases, where the range of $\Delta \chi$ varies from $0.02 \mathrm{eV}$ to $1.59 \mathrm{eV}$, the $\mathrm{Ti}_{2} \mathrm{Ni}$ structure has also shown softening: hardness decreases as the composition 


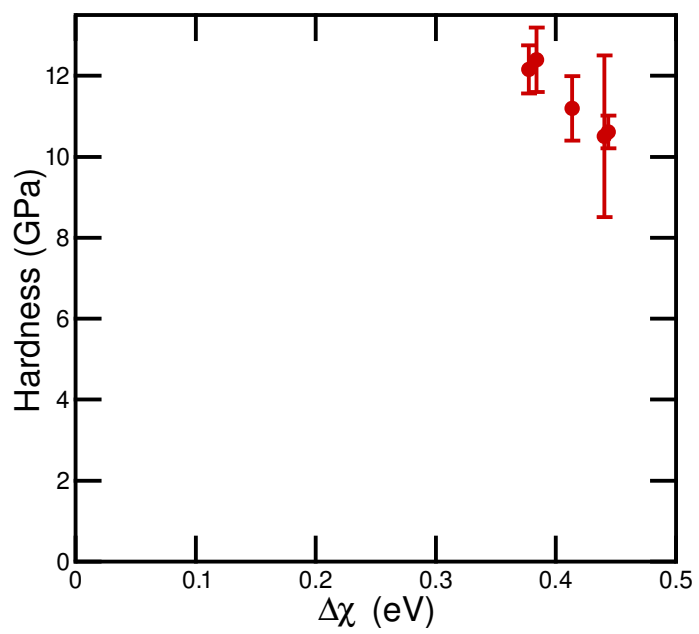

Figure 5: The variation of the hardness with electronegativity difference across the $\{111\}$ planes for alloys with the $\mathrm{Ti}_{2} \mathrm{Ni}$ structure: $\mathrm{Ti}_{2} \mathrm{Ni}, \mathrm{Ti}_{2} \mathrm{Co}, \mathrm{Hf}_{2} \mathrm{Co}, \mathrm{Ti}_{2}(\mathrm{Co}, \mathrm{Ni})$, and (Hf, $\left.\mathrm{Ti}\right)_{2} \mathrm{Co}$. From [70].

is varied to increase the chemical, and thus elastic, heterogeneity within the unit cell. This demonstrates the effect of heterogeneity softening in a cubic structure. Cubic materials are not limited to a small number of slip systems like the hexagonal MAX phases, suggesting that sub-unit cell elastic heterogeneity offers a general route to tailoring the yield stress, and thereby the toughness, of non-metallic materials.

\section{Summary and conclusions}

A number of ductility criteria have been discussed, particularly the widely used Pugh criterion. These ductility criteria are usually based on properties that are obtained easily by measurement or calculation, such as macroscopic elastic constants or the stacking fault energy. They give a general trend within a class of materials for which the mechanism of deformation remains the same, which is not surprising, given that the ease of slip must be linked to properties like the shear modulus.

However, these criteria have significant shortfalls, for example they cannot explain observations such as the effects of size. The implicit assumptions inherent in these criteria, such as the nature of plastic deformation and the effects that crystal structure have upon it, limit the transferability of ductility criteria across classes of materials, and in turn the predictive power of such criteria. In particular the effects of crystal structure on deformation processes are not accounted for. For example, the criteria do not explain the lack of ductility in the Laves phases, instead many of these criteria predict ductile behaviour. Additionally, due to the effects of sub-unit cell heterogeneity, the criteria do not capture the easy plastic flow observed in the MAX phases 305 or the reduced lattice resistance of the $\mathrm{Ti}_{2} \mathrm{Ni}$ structure.

Such effects require a full treatment including an appropriate model of the barrier to dislocation motion, such as the Peierls model. Hence there do not seem to be short cuts to finding novel phases with attractive properties, since as yet unforeseen effects will undoubtedly break some implicit assumption of any simple 
criterion.

\section{Acknowledgements}

This work was supported by the EPSRC/Rolls-Royce plc Strategic Partnership in structural systems for gas turbines (EP/H022309/1).

\section{References}

[1] S. F. Pugh, XCII. relations between the elastic moduli and the plastic properties of polycrystalline pure metals, The London, Edinburgh, and Dublin Philosophical Magazine and Journal of Science 45 (1954) 823-843.

[2] F. C. Frank, W. T. Read, Multiplication processes for slow moving dislocations, Physical Review 79 (1950) 722-723.

[3] A. A. Griffith, The phenomena of rupture and flow in solids, Philosophical Transactions of the Royal Society A: Mathematical, Physical and Engineering Sciences 221 (1921) 163-198.

[4] J. W. Obreimoff, The splitting strength of mica, Proceedings of the Royal Society of London. Series A, Containing Papers of a Mathematical and Physical Character 127 (1930) 290-297.

[5] H. A. Elliott, An analysis of the conditions for rupture due to Griffith cracks, Proceedings of the Physical Society 59 (1947) 208-223.

[6] T. Suzuki, Y. Kamimura, H. O. K. Kirchner, Plastic homology of bcc metals, Philosophical Magazine A 79 (1999) 1629-1642.

[7] H. Frost, M. Ashby, Deformation-mechanism maps: the plasticity and creep of metals and ceramics, Pergamon Press, 1982.

[8] H. M. Ledbetter, R. P. Reed, Elastic Properties of Metals and Alloys, I. Iron, Nickel, and Iron-Nickel Alloys, Journal of Physical and Chemical Reference Data 2 (1973) 531-618.

[9] D. Tabor, The Hardness of Metals, Monographs on the physics and chemistry of materials, OUP, Oxford, 2000.

[10] J. W. Nash, H. R. Ogden, R. E. Durtschi, I. E. Campbell, Preparation and properties of iodide vanadium, Journal of The Electrochemical Society 100 (1953) 272.

335 [11] Metals Handbook, American Society for Metals, 1948.

[12] F. R. N. Nabarro, Mathematical theory of stationary dislocations, Advances in Physics 1 (1952) 269-394. 
[13] J. P. Hirth, J. Lothe, Effects of solute atoms on dislocation motion, in: Theory of Dislocations, second ed., John Wiley \& Sons, Ltd, New York, 1982, pp. 584-633.

[14] J. J. Gilman, Shear modulus, in: Electronic Basis of the Strength of Materials, Cambridge University Press, Cambridge, 2001, pp. 142-173. doi:10.1017/СB09780511541247.015.

[15] P. Panfilov, A. Yermakov, Brittle intercrystalline fracture in iridium, Platinum Metals Review 45 (2001) 179-183.

[16] P. Panfilov, A. Yermakov, On brittle fracture in polycrystalline iridium, Journal of Materials Science 39 (2004) 4543-4552.

[17] D. G. Pettifor, Theoretical predictions of structure and related properties of intermetallics, Materials Science and Technology 8 (1992) 345-349.

[18] H. Niu, X.-Q. Chen, P. Liu, W. Xing, X. Cheng, D. Li, Y. Li, Extra-electron induced covalent strengthening and generalization of intrinsic ductile-to-brittle criterion, Scientific Reports 2 (2012) 718.

[19] H. Xing, A. Dong, J. Huang, J. Zhang, B. Sun, Revisiting intrinsic brittleness and deformation behavior of B2 NiAl intermetallic compound: A first-principles study, Journal of Materials Science \& Technology (2017).

[20] H. Hu, X. Wu, R. Wang, Z. Jia, W. Li, Q. Liu, Structural stability, mechanical properties and stacking fault energies of $\mathrm{TiAl}_{3}$ alloyed with zn, cu, ag: First-principles study, Journal of Alloys and Compounds 666 (2016) 185-196.

[21] K. A. Gschneidner, Physical properties and interrelationships of metallic and semimetallic elements, in: Solid State Physics - Advances in Research and Applications, volume 16, 1964, pp. 275-426. doi:10. 1016/S0081-1947(08)60518-4.

[22] A. G. Every, A. K. McCurdy, Table 5. Cubic system. Intermetallic compounds: Datasheet from LandoltBörnstein - Group III Condensed Matter · Volume 29A: "Second and Higher Order Elastic Constants" SpringerMaterials, 1992. doi:10.1007/10046537_12.

[23] M. Ozawa, M. Yoshizawa, H. Sugawara, Y. Ônuki, Elastic constants of $\mathrm{LaRh}_{2}$ and $\mathrm{CeRh}_{2}$, $\mathrm{Physica} \mathrm{B}$ : Condensed Matter 206-207 (1995) 267-269.

[24] D. J. J. Thoma, F. Chu, P. Peralta, P. G. G. Kotula, K. C. C. Chen, T. E. E. Mitchell, Elastic and mechanical properties of $\mathrm{Nb}(\mathrm{Cr}, \mathrm{V})_{2} \mathrm{C} 15$ laves phases, Materials Science and Engineering: A 239-240 (1997) 251-259.

[25] M. Yoshizawa, M. Tamura, M. Ozawa, D.-H. Yoon, H. Sugawara, H. Sato, Y. nuki, Static and Dynamic Properties of the Vortices in Single Crystalline $\mathrm{CeRu}_{2}$, Journal of the Physical Society of Japan 66 (1997) 2355-2366. 
[26] S. Hong, C. Fu, M. Yoo, Elastic properties and stacking fault energies of $\mathrm{Cr}_{2} \mathrm{Ta}$, Intermetallics 7 (1999) $1169-1172$.

[27] M. Yoshizawa, T. Sakashita, N. Yoshimoto, M. Nakamura, G. Bruls, B. Lthi, H. Sugawara, H. Sato, Elastic anomalies and acoustic quantum oscillation in $\mathrm{CeCo}_{2}$, Physica B: Condensed Matter 281-282 (2000) $740-741$.

[28] B. Mayer, H. Anton, E. Bott, M. Methfessel, J. Sticht, J. Harris, P. C. Schmidt, Ab-initio calculation of the elastic constants and thermal expansion coefficients of Laves phases, Intermetallics 11 (2003) 23-32.

[29] X. Tao, Y. Ouyang, H. Liu, F. Zeng, Y. Feng, Y. Du, Z. Jin, Ab initio calculation of the total energy and elastic properties of Laves phase $\mathrm{C} 15 \mathrm{Al}_{2} \mathrm{RE}$ ( $\mathrm{re}=\mathrm{sc}$, y, la, celu), Computational Materials Science 44 (2008) 392-399.

[30] S. Ganeshan, S. Shang, H. Zhang, Y. Wang, M. Mantina, Z. Liu, Elastic constants of binary Mg compounds from first-principles calculations, Intermetallics 17 (2009) 313-318.

[31] A. Yakoubi, O. Baraka, B. Bouhafs, Structural and electronic properties of the Laves phase based on rare earth type $\mathrm{BaM}_{2}(\mathrm{M}=\mathrm{Rh}, \mathrm{Pd}, \mathrm{Pt})$, Results in Physics 2 (2012) 58-65.

[32] T. Chihi, M. Fatmi, B. Ghebouli, Ab initio calculations for properties of Laves phase $\mathrm{V}_{2} \mathrm{M}(\mathrm{M}=\mathrm{Zr}$, Hf, Ta) compounds, American Journal of Modern Physics 2 (2013) 88.

[33] S. Chen, Y. Sun, Y.-H. Duan, B. Huang, M.-J. Peng, Phase stability, structural and elastic properties of C15-type Laves transition-metal compounds $\mathrm{MCo}_{2}$ from first-principles calculations, Journal of Alloys and Compounds 630 (2015) 202-208.

[34] S. Korte, W. J. Clegg, Studying plasticity in hard and soft Nb-Co intermetallics, Advanced Engineering Materials 14 (2012) 991-997.

[35] A. Von Keitz, G. Sauthoff, Laves phases for high temperatures-Part II: Stability and mechanical properties, Intermetallics 10 (2002) 497-510.

[36] T. Ohta, Y. Nakagawa, Y. Kaneno, Microstructures and mechanical properties of $\mathrm{NbCr}_{2}$ and $\mathrm{ZrCr}_{2}$ Laves phase alloys prepared by powder metallurgy, Journal of Materials Science 8 (2003) 657-665.

[37] N. Bouras, M. Madjoubi, M. Kolli, S. Benterki, M. Hamidouche, Thermal and mechanical characterization of borosilicate glass, Physics Procedia 2 (2009) 1135-1140.

[38] G. A. Gogotsi, Fracture toughness of ceramics and ceramic composites, Ceramics International 29 (2003) $777-784$.

[39] F. Östlund, P. R. Howie, R. Ghisleni, S. Korte, K. Leifer, W. J. Clegg, J. Michler, Ductile-brittle transition in micropillar compression of GaAs at room temperature, Philosophical Magazine 91 (2011) 1190-1199. 
[40] S. Korte, W. J. Clegg, Discussion of the dependence of the effect of size on the yield stress in hard materials studied by microcompression of MgO, Philosophical Magazine 91 (2011) 1150-1162.

[41] S. Korte, J. S. Barnard, R. J. Stearn, W. J. Clegg, Deformation of silicon - Insights from microcompression testing at $25-500{ }^{\circ} \mathrm{C}$, International Journal of Plasticity 27 (2011) 1853-1866.

[42] A. C. Fischer-Cripps, Nanoindentation, Mechanical Engineering Series, Springer, New York, NY, 2011. doi:10.1007/978-1-4419-9872-9.

[43] J. R. Rice, R. Thomson, Ductile versus brittle behaviour of crystals, Philosophical Magazine 29 (1974) 73-97.

[44] A. Kelly, W. R. Tyson, A. H. Cottrell, Ductile and brittle crystals, Philosophical Magazine 15 (1967) $567-586$.

[45] A. Cottrell, Surprises in materials science, Interdisciplinary Science Reviews 22 (1997) 318-324.

[46] J. R. Rice, Dislocation nucleation from a crack tip: An analysis based on the Peierls concept, Journal of the Mechanics and Physics of Solids 40 (1992) 239-271.

[47] S. J. Zhou, A. E. Carlsson, R. Thomson, Crack blunting effects on dislocation emission from cracks, Physical Review Letters 72 (1994) 852-855.

[48] D. L. Price, B. R. Cooper, J. M. Wills, Full-potential linear-muffin-tin-orbital study of brittle fracture in titanium carbide, Physical Review B 46 (1992) 11368-11375.

[49] R. Yu, L. L. He, H. Q. Ye, Effects of si and al on twin boundary energy of TiC, Acta Materialia 51 (2003) 2477-2484.

[50] N. I. Medvedeva, A. N. Enyashin, A. L. Ivanovskii, Modeling of the electronic structure, chemical bonding, and properties of ternary silicon carbide $\mathrm{Ti}_{3} \mathrm{SiC}_{2}$, Journal of Structural Chemistry 52 (2011) $785-802$.

[51] A.-S. Farle, C. Kwakernaak, S. van der Zwaag, W. G. Sloof, A conceptual study into the potential of $\mathrm{M}_{n+1} \mathrm{AX}_{n}$-phase ceramics for self-healing of crack damage, Journal of the European Ceramic Society 35 (2015) 37-45.

[52] D. B. Miracle, H. A. Lipsitt, Mechanical properties of fine-grained substoichiomebic titanium carbide, Journal of the American Ceramic Society 66 (1983) 592-597.

[53] M. W. Barsoum, T. El-Raghy, Room-temperature ductile carbides, Metallurgical and Materials Transactions A 30 (1999) 363-369.

${ }_{430}^{454]}$ C. Humphrey, Deformation in MAX Phases, PhD Thesis, University of Cambridge, 2012. 
[55] P. B. Hirsch, Dislocations in semiconductors, Materials Science and Technology 1 (1985) 666-677.

[56] R. Peierls, The size of a dislocation, Proceedings of the Physical Society 52 (1940) 34-37.

[57] R. Telle, A. Momozawa, D. Music, J. M. Schneider, Boride-based nano-laminates with MAX-phase-like behaviour, Journal of Solid State Chemistry 179 (2006) 2850-2857.

${ }_{435}$ [58] M. Sygnatowicz, R. A. Cutler, D. K. Shetty, $\zeta-\mathrm{Ta}_{4} \mathrm{C}_{3-x}$ : A high fracture toughness carbide with rising-crack-growth-resistance (R-curve) behavior, Journal of the American Ceramic Society 98 (2015) 2601-2608.

[59] Z. M. Sun, Progress in research and development on MAX phases: a family of layered ternary compounds, International Materials Reviews 56 (2011) 143-166.

${ }_{440}$ [60] A. Schallamach, How does rubber slide?, Wear 17 (1971) 301-312.

[61] A. Kushima, X. Qian, P. Zhao, S. Zhang, J. Li, Ripplocations in van der Waals layers, Nano Letters 15 (2015) 1302-1308.

[62] J. Gruber, A. . Lang, J. Griggs, M. L. Taheri, G. J. Tucker, M. W. Barsoum, Evidence for bulk ripplocations in layered solids, Scientific Reports 6 (2016) 33451.

${ }_{445}$ [63] J. P. Hirth, J. Lothe, The theory of straight dislocations, in: Theory of Dislocations, second ed., John Wiley \& Sons, Ltd, New York, 1982, pp. 58-90.

[64] F. R. N. Nabarro, Stationary dislocations in a crystal, in: Theory of crystal dislocations, Dover Books on Physics and Chemistry, Dover Publications, New York, 1987, pp. 120-154.

[65] M. J. Hÿtch, J.-L. Putaux, J.-M. Pénisson, Measurement of the displacement field of dislocations to $0.03 \AA$ by electron microscopy, Nature 423 (2003) 270-273.

[66] J. P. Hirth, J. Lothe, The influence of lattice periodicity, in: Theory of Dislocations, second ed., John Wiley \& Sons, Ltd, New York, 1982, pp. 201-250.

[67] D. Music, J. M. Schneider, Elastic properties of $\operatorname{Sr}_{n+1} \operatorname{Ti}_{n} \mathrm{O}_{3 n+1}$ phases $(n=1--3, \infty)$, Journal of Physics: Condensed Matter 20 (2008) 055224.

[68] J. Emmerlich, D. Music, M. Braun, P. Fayek, F. Munnik, J. M. Schneider, A proposal for an unusually stiff and moderately ductile hard coating material: $\mathrm{Mo}_{2} \mathrm{BC}$, Journal of Physics D: Applied Physics 42 (2009) 185406.

[69] K. Gouriet, P. Carrez, P. Cordier, A. Guitton, A. Joulain, L. Thilly, C. Tromas, Dislocation modelling in $\mathrm{Ti}_{2}$ AlN max phase based on the Peierls-Nabarro model, Philosophical Magazine 95 (2015) 2539-2552. 
[70] P. R. Howie, R. P. Thompson, S. Korte-Kerzel, W. J. Clegg, Softening non-metallic crystals by inhomogeneous elasticity, Scientific Reports 7 (2017) 11602.

[71] M. Magnuson, M. Mattesini, Chemical bonding and electronic-structure in max phases as viewed by x-ray spectroscopy and density functional theory, Thin Solid Films 621 (2017) 108-130.

[72] D. Hull, T. W. Clyne, Elastic deformation of long-fibre composites, in: D. R. Clarke, S. Suresh, I. M. Ward (Eds.), An Introduction to Composite Materials, Cambridge Solid State Science Series, second ed., Cambridge University Press, Cambridge, 1996, pp. 60-77. doi:10.1017/CB09781139170130.006.

[73] W. J. Clegg, L. J. Vandeperre, J. E. Pitchford, Energy changes and the lattice resistance, Key Engineering Materials 317-318 (2006) 271-276.

[74] A. H. Cottrell, Dislocations in crystals, in: R. Fowler, P. Kapitzer, N. Mott, E. Bullard (Eds.), Dislocations and plastic flow in crystals, Oxford University Press, 1953, pp. 58-98.

[75] G. A. Yurko, J. W. Barton, J. G. Parr, The crystal structure of $\mathrm{Ti}_{2} \mathrm{Ni}$, Acta Crystallographica 12 (1959) 909-911.

[76] G. A. Yurko, J. W. Barton, J. G. Parr, The crystal structure of $\mathrm{Ti}_{2} \mathrm{Ni}$ (a correction), Acta Crystallographica 15 (1962) 1309.

[77] Inorganic crystal structure database - ICSD, URL: icsd.cds.rsc.org, 2017. Accessed December 2017.

[78] W. Steurer, Stable clusters in quasicrystals: fact or fiction?, Philosophical Magazine 86 (2006) 11051113.

[79] N. Ivanović, D. Rodić, V. Koteski, I. Radisavljević, N. Novaković, D. Marjanović, M. Manasijević, S. Koički, Cluster approach to the $\mathrm{Ti}_{2} \mathrm{Ni}$ structure type, Acta Crystallographica Section B Structural Science 62 (2006) 1-8.

[80] F. Stein, M. Palm, G. Sauthoff, Structure and stability of Laves phases. Part I. Critical assessment of factors controlling Laves phase stability, Intermetallics 12 (2004) 713-720.

[81] F. Stein, M. Palm, G. Sauthoff, Structure and stability of Laves phases part II — structure type variations in binary and ternary systems, Intermetallics 13 (2005) 1056-1074.

[82] R. P. Thompson, Plastic Deformation in Complex Crystal Structures, PhD Thesis, University Of Cambridge, 2017.

[83] N. Moulay, H. Rached, M. Rabah, S. Benalia, D. Rached, A. H. Reshak, N. Benkhettou, P. Ruterana, First-principles calculations of the elastic, and electronic properties of $\mathrm{YFe}_{2}, \mathrm{NiFe}_{2}$ and $\mathrm{YNiFe}_{4}$ intermetallic compounds, Computational Materials Science 73 (2013) 56-64. 

loading axis, rather than $25^{\circ}$, the angle between the slip plane normal and the loading axis.

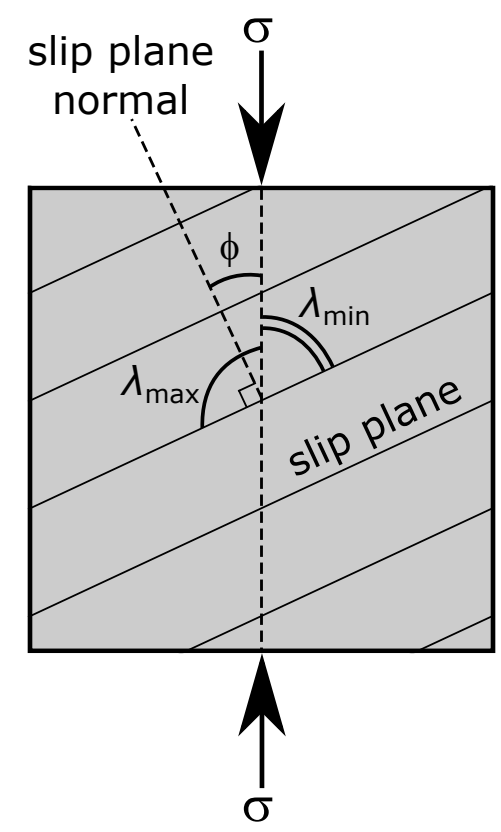

Figure A.6: Schematic showing the geometry of the Schmid factor calculation. The angle between the slip plane normal and the loading direction, $\phi$, is given by Barsoum and El-Raghy [53] to be $25^{\circ}$. The angle between the slip direction and the loading axis, $\lambda$, is unknown and will vary between grains, but has a maximum value of $115^{\circ}$, i.e. $\lambda_{\max }=90+25^{\circ}$, and a minimum value of $65^{\circ}$, i.e. $\lambda_{\min }=90-25^{\circ}$. 
Data used in Figure 1

\begin{tabular}{|l|c|c|c|c|c|c|c|c|}
\hline Element & $C_{11}(\mathrm{GPa})$ & $C_{12}(\mathrm{GPa})$ & $C_{44}(\mathrm{GPa})$ & $G(\mathrm{GPa})$ & $K(\mathrm{GPa})$ & $E(\mathrm{GPa})$ & $G / K$ & $\frac{C_{12}-C_{44}}{E}$ \\
\hline $\mathrm{Pb}$ & 48.8 & 41.4 & 14.8 & 14.8 & 43.5 & 16.0 & 0.34 & 1.69 \\
$\mathrm{Al}$ & 108.2 & 62.2 & 28.4 & 28.4 & 76.8 & 72.4 & 0.37 & 0.48 \\
$\mathrm{Th}$ & 77.0 & 48.9 & 47.8 & 47.8 & 58.3 & 76.0 & 0.82 & 0.01 \\
$\mathrm{Au}$ & 192.9 & 163.8 & 41.5 & 41.5 & 172.9 & 79.4 & 0.24 & 1.57 \\
$\mathrm{Ag}$ & 122.0 & 92.0 & 45.5 & 45.5 & 101.1 & 82.2 & 0.45 & 0.58 \\
$\mathrm{Cu}$ & 169.0 & 122.0 & 75.3 & 75.3 & 136.9 & 126.0 & 0.55 & 0.38 \\
$\mathrm{Pd}$ & 221.0 & 171.0 & 70.8 & 70.8 & 186.3 & 126.0 & 0.38 & 0.81 \\
$\mathrm{Pt}$ & 347.0 & 251.0 & 76.5 & 76.5 & 283.3 & 174.0 & 0.27 & 1.02 \\
$\mathrm{Ni}$ & 247.0 & 153.0 & 122.0 & 122.0 & 184.8 & 197.0 & 0.66 & 0.16 \\
$\mathrm{Rh}$ & 413.0 & 194.0 & 184.0 & 184.0 & 266.7 & 380.0 & 0.69 & 0.02 \\
$\mathrm{Ir}$ & 580.0 & 260.0 & 270.0 & 270.0 & 364.9 & 538.0 & 0.74 & -0.02 \\
\hline
\end{tabular}

Table A.1: Elastic data for the f.c.c. metals used to plot Figure 1. Data are from [14] and [21]. 


\begin{tabular}{|c|c|c|c|c|c|c|c|c|c|c|}
\hline Phase & $C_{11}(\mathrm{GPa})$ & $C_{12}(\mathrm{GPa})$ & $C_{44}(\mathrm{GPa})$ & $\mathrm{G}(\mathrm{GPa})$ & $\mathrm{B}(\mathrm{GPa})$ & $\mathrm{E}(\mathrm{GPa})$ & $\frac{C_{12}-C_{44}}{E}$ & $G / K$ & $\mathrm{E}$ or $\mathrm{C}^{*}$ & Ref \\
\hline $\mathrm{CeRu}_{2}$ & 138.0 & 129.0 & 19.0 & 4.5 & 132.0 & 13.3 & 8.241 & 0.034 & $\mathrm{E}$ & {$[25]$} \\
\hline $\mathrm{ZrV}_{2}$ & 154.1 & 128.6 & 9.2 & 9.2 & 137.1 & 37.1 & 3.222 & 0.067 & $\mathrm{C}$ & [32] \\
\hline $\mathrm{HfV}_{2}$ & 128.0 & 102.0 & 17.0 & 13.0 & 110.7 & 37.5 & 2.265 & 0.117 & $\mathrm{E}$ & {$[22]$} \\
\hline $\mathrm{TaV}_{2}$ & 240.9 & 169.2 & 28.6 & 28.6 & 193.1 & 101.3 & 1.387 & 0.148 & $\mathrm{C}$ & {$[32]$} \\
\hline $\mathrm{BaRh}_{2}$ & 158.5 & 113.3 & 35.1 & 22.6 & 128.4 & 63.9 & 1.223 & 0.176 & $\mathrm{C}$ & [31] \\
\hline $\mathrm{BaPd}_{2}$ & 137.0 & 91.6 & 46.2 & 22.7 & 106.8 & 63.6 & 0.714 & 0.213 & $\mathrm{C}$ & [31] \\
\hline $\mathrm{YCo}_{2}$ & 185.4 & 118.5 & 48.9 & 33.5 & 140.8 & 93.0 & 0.748 & 0.238 & $\mathrm{C}$ & [33] \\
\hline $\mathrm{YFe}_{2}$ & 205.9 & 131.5 & 49.6 & 37.2 & 156.3 & 103.3 & 0.794 & 0.238 & $\mathrm{C}$ & [83] \\
\hline $\mathrm{ScCo}_{2}$ & 206.3 & 131.1 & 67.3 & 37.6 & 156.2 & 104.4 & 0.611 & 0.241 & $\mathrm{C}$ & [33] \\
\hline $\mathrm{HfCr}_{2}$ & 201.0 & 126.0 & 46.0 & 37.5 & 151.0 & 103.9 & 0.770 & 0.248 & $\mathrm{C}$ & {$[26]$} \\
\hline $\mathrm{CeCo}_{2}$ & 113.5 & 69.5 & 27.3 & 22.0 & 84.2 & 60.7 & 0.695 & 0.261 & $\mathrm{E}$ & {$[27]$} \\
\hline $\mathrm{HoCo}_{2}$ & 153.0 & 93.0 & 40.1 & 30.0 & 113.0 & 82.7 & 0.640 & 0.265 & $\mathrm{E}$ & {$[22]$} \\
\hline $\mathrm{LaRh}_{2}$ & 140.0 & 84.0 & 48.0 & 28.0 & 102.7 & 77.0 & 0.468 & 0.273 & $\mathrm{E}$ & [23] \\
\hline $\mathrm{UCo}_{2}$ & 219.0 & 127.0 & 65.4 & 46.0 & 157.7 & 125.8 & 0.490 & 0.292 & $\mathrm{E}$ & {$[22]$} \\
\hline $\mathrm{CeRh}_{2}$ & 146.0 & 84.0 & 58.0 & 31.0 & 104.7 & 84.6 & 0.307 & 0.296 & $\mathrm{E}$ & [23] \\
\hline $\mathrm{MgCu}_{2}$ & 123.0 & 70.6 & 41.2 & 26.2 & 88.1 & 71.5 & 0.411 & 0.298 & $\mathrm{E}$ & {$[22]$} \\
\hline $\mathrm{BaPt}_{2}$ & 198.8 & 112.0 & 60.4 & 43.4 & 140.9 & 118.0 & 0.438 & 0.308 & $\mathrm{C}$ & [31] \\
\hline $\mathrm{TaCr}_{2}$ & 342.0 & 192.0 & 86.0 & 75.0 & 242.0 & 203.9 & 0.520 & 0.310 & $\mathrm{C}$ & [28] \\
\hline $\mathrm{ZrCr}_{2}$ & 255.0 & 141.0 & 58.0 & 57.0 & 179.0 & 154.6 & 0.537 & 0.318 & $\mathrm{C}$ & [28] \\
\hline $\mathrm{ZrCo}_{2}$ & 233.0 & 113.0 & 83.7 & 60.0 & 153.0 & 159.2 & 0.184 & 0.392 & $\mathrm{E}$ & {$[22]$} \\
\hline $\mathrm{HfCo}_{2}$ & 256.0 & 123.0 & 90.3 & 66.5 & 167.3 & 176.2 & 0.186 & 0.397 & $\mathrm{E}$ & {$[22]$} \\
\hline $\mathrm{NbCo}_{2}$ & 349.8 & 162.3 & 130.6 & 93.8 & 224.8 & 246.9 & 0.128 & 0.417 & $\mathrm{C}$ & [33] \\
\hline $\mathrm{NbCr}_{2}$ & 301.7 & 138.3 & 81.7 & 81.7 & 192.7 & 214.8 & 0.263 & 0.424 & $\mathrm{E}$ & {$[24]$} \\
\hline $\mathrm{TiCo}_{2}$ & 324.7 & 148.2 & 130.4 & 88.3 & 207.0 & 231.8 & 0.077 & 0.426 & $\mathrm{C}$ & [33] \\
\hline $\mathrm{TaCo}_{2}$ & 383.5 & 173.4 & 143.9 & 105.1 & 243.4 & 275.5 & 0.107 & 0.432 & $\mathrm{C}$ & [33] \\
\hline $\mathrm{TiCr}_{2}$ & 322.0 & 140.0 & 99.0 & 91.0 & 200.7 & 237.2 & 0.173 & 0.453 & $\mathrm{C}$ & [28] \\
\hline $\mathrm{LaMg}_{2}$ & 58.4 & 24.9 & 21.8 & 16.8 & 36.1 & 43.5 & 0.071 & 0.464 & $\mathrm{C}$ & [30] \\
\hline $\mathrm{NdAl}_{2}$ & 141.0 & 47.0 & 42.8 & 42.8 & 78.3 & 117.5 & 0.036 & 0.546 & $\mathrm{E}$ & {$[22]$} \\
\hline $\mathrm{PrAl}_{2}$ & 138.0 & 41.8 & 45.2 & 45.2 & 73.9 & 118.6 & -0.029 & 0.612 & $\mathrm{E}$ & {$[22]$} \\
\hline $\mathrm{LaAl}_{2}$ & 143.7 & 32.0 & 43.0 & 43.0 & 69.2 & 132.0 & -0.083 & 0.621 & $\mathrm{E}$ & {$[22]$} \\
\hline $\mathrm{CeAl}_{2}$ & 146.6 & 29.8 & 43.7 & 43.7 & 68.7 & 136.5 & -0.102 & 0.636 & $\mathrm{E}$ & {$[22]$} \\
\hline $\mathrm{SmAl}_{2}$ & 155.8 & 34.7 & 48.4 & 48.4 & 75.1 & 143.1 & -0.095 & 0.644 & $\mathrm{C}$ & [29] \\
\hline $\mathrm{LuAl}_{2}$ & 174.3 & 38.1 & 54.9 & 54.9 & 83.5 & 160.7 & -0.105 & 0.658 & $\mathrm{C}$ & [29] \\
\hline $\mathrm{UAl}_{2}$ & 170.4 & 39.2 & 54.8 & 54.8 & 82.9 & 155.7 & -0.100 & 0.661 & $\mathrm{E}$ & {$[22]$} \\
\hline $\mathrm{DyAl}_{2}$ & 168.2 & 36.1 & 53.0 & 53.0 & 80.1 & 155.4 & -0.109 & 0.662 & $\mathrm{C}$ & [29] \\
\hline $\mathrm{HoAl}_{2}$ & 170.2 & 36.6 & 53.7 & 53.7 & 81.1 & 157.3 & -0.109 & 0.662 & $\mathrm{C}$ & [29] \\
\hline $\mathrm{ErAl}_{2}$ & 171.9 & 36.9 & 54.3 & 54.3 & 81.9 & 158.8 & -0.109 & 0.663 & $\mathrm{C}$ & [29] \\
\hline $\mathrm{YAl}_{2}$ & 170.8 & 34.0 & 56.2 & 56.2 & 79.6 & 159.5 & -0.139 & 0.706 & $\mathrm{E}$ & {$[22]$} \\
\hline $\mathrm{TmAl}_{2}$ & 177.8 & 33.2 & 59.0 & 59.0 & 81.4 & 167.4 & -0.154 & 0.725 & $\mathrm{E}$ & {$[22]$} \\
\hline $\mathrm{ScAl}_{2}$ & 214.0 & 36.0 & 72.0 & 72.0 & 95.3 & 203.6 & -0.177 & 0.755 & $\mathrm{C}$ & [28] \\
\hline $\mathrm{GdAl}_{2}$ & 160.5 & 36.8 & 59.5 & 59.5 & 78.0 & 146.8 & -0.155 & 0.762 & $\mathrm{E}$ & {$[22]$} \\
\hline $\mathrm{CaAl}_{2}$ & 97.0 & 22.4 & 36.6 & 36.6 & 47.3 & 88.6 & -0.160 & 0.774 & $\mathrm{E}$ & {$[22]$} \\
\hline $\mathrm{TbAl}_{2}$ & 144.0 & 33.0 & 68.0 & 55.5 & 70.0 & 131.7 & -0.266 & 0.793 & $\mathrm{E}$ & {$[22]$} \\
\hline $\mathrm{EuAl}_{2}$ & 104.7 & 23.9 & 40.7 & 40.4 & 50.8 & 95.8 & -0.175 & 0.795 & $\mathrm{E}$ & {$[22]$} \\
\hline $\mathrm{YbAl}_{2}$ & 114.4 & 13.0 & 46.8 & 46.8 & 46.8 & 111.7 & -0.302 & 1.000 & $\mathrm{E}$ & {$[22]$} \\
\hline
\end{tabular}

Table A.2: Laves Phase elastic data plotted in Figure 1. ${ }^{*}$ indicates experimental vs calculated values. 